\title{
Uso de sistemas de control de versiones para aplicar estrategias de evaluación por pares en contextos tecnológicos Using Version Control Systems to apply peer review techniques in engineering education
}

\author{
Departamento de Ingenierías Mecánica, \\ Informática y Aeroespacial \\ Universidad de León \\ León, España
}

Ángel M. Guerrero-Higueras, Miguel Á. Conde, Vicente Matellán

am.guerrero@unileon.es,miguel.conde@unileon.es, vicente.matellan@unileon.es

\begin{abstract}
Resumen- Diferentes metodologías educativas han demostrado que un aspecto muy positivo para mejorar el aprendizaje del estudiante es que éste sea parte central del mismo, y especialmente que se involucre de manera activa en los procesos de aprendizaje. En este sentido la aplicación de técnicas de evaluación por pares ha sido una aproximación muy popular. Sin embargo, en enseñanzas de carácter ingenieril y especialmente enseñanzas técnicas, las actividades a evaluar implican en muchas ocasiones el uso de lenguajes o herramientas muy específicas. Esto hace que la evaluación por pares sea más compleja e implique que tanto alumnos como profesores tengan que utilizar diferentes contextos para la evaluación (como una herramienta de desarrollo software y una plataforma de aprendizaje). De cara a solventar este problema el presente trabajo propone el uso de sistemas de control de versiones que van a permitir almacenar los resultados obtenidos e interactuar al responsable del trabajo con sus revisores. En concreto, en este artículo se presenta la aplicación de técnicas de evaluación por pares en un grupo de 46 alumnos. Los resultados muestran que los discentes que usan activamente la herramienta con fines de evaluación tienen mejores resultados asociados.
\end{abstract}

Palabras clave: Evaluación por pares, Sistemas de Control de Versiones, Enseñanzas Técnicas, Interacción, Colaboración

Abstract- Different studies have shown that a very positive factor to improve students learning is that they were the center of teaching and learning processes and also to be an active part in them. In this sense, the application of peer review techniques is a very popular approach. However, in engineering education and special in technical degrees the activities to assess consist of the use of very specific tools and languages. This makes peer evaluation more complex in this context than in others. It requires that both teachers and students use different tools and platforms to complete the evaluation. In order to solve this, the present work aims to apply a version control system to facilitate manage different results versions and also to interact with reviewers in the peer review process. In this specific work, the authors present a case study with 46 students that employ a version control system to apply peer review. Results show that students that use properly the tool have better performance.

Keywords: Peer review, Version Control Systems, Technical Degrees, Interaction, Collaboration

\section{INTRODUCCIÓN}

Los procesos educativos tienen como cometido formar como personas y profesionales a los individuos para que estos puedan desempeñarse de forma exitosa en su vida diaria. Con esta idea se ha planteado el uso de diferentes metodologías, herramientas, etc. En este sentido unas de las más representativas pueden ser las Tecnologías de la Información y la Comunicación (TIC) que facilitan la implementación de estas opciones. Por ejemplo, las TIC van a posibilitar que pueda aplicarse un aprendizaje centrado en el estudiante incluso en grupos grandes (O’Neill \& McMahon, 2005), que se materialicen conceptos como el de aprendizaje masivo (Pappano, 2012), o que puedan aplicarse planteamientos como el de aprendizaje basado en problemas (Savery \& Duffy, 1995).

Las TIC también aportan facilidades para mejorar aspectos concretos de los procesos educativos como es la evaluación. Al popularizarse herramientas como las plataformas de aprendizaje es posible recopilar gran cantidad de evidencias de la actividad del usuario, con lo que es factible llevar a cabo análisis exhaustivos de lo que está ocurriendo en un contexto determinado y definir acciones (bien en el momento o al finalizar la actividad educativa) que permitan corregir los posibles problemas encontrados. En ese sentido la Analítica de Aprendizaje, también conocida como Learning Analytics, tiene una especial relevancia (Conde \& Hernández-García, 2015; Hernández-García \& Conde, 2014).

Pero en el contexto de la evaluación, las TIC no solo facilitan la recogida y análisis de evidencias, sino también la aplicación de técnicas que en grupos grandes podrían además ser muy tediosas. Un ejemplo en este sentido puede ser la evaluación por pares, coevaluación o peer review. La evaluación por pares es un método utilizado con éxito durante décadas en diferentes contextos. En concreto implica un aprendizaje colaborativo en el que unos estudiantes evalúan el trabajo de otros y les proporcionan retroalimentación (Pearce, Mulder, \& Baik, 2005; Topping, Smith, Swanson, \& Elliot, 2000; van den Berg, Admiraal, \& Pilot, 2006).

El presente trabajo se centra en la aplicación de este tipo de metodologías de evaluación mediante el uso de las TIC en 
entornos de enseñanza de tecnología. En estos contextos, el concepto de evaluación varía con respecto a otros ámbitos. Se trata de titulaciones eminentemente prácticas, dónde la mayor parte de la evaluación debería basarse en actividades o problemas en lugar de exámenes meramente teóricos (Felder \& Silverman, 1988). Dada esta situación, no es lo mismo evaluar un trabajo teórico que la entrega de una actividad práctica con múltiples soluciones válidas. De ahí que la aplicación de herramientas tecnológicas vaya a facilitar tanto gestión de la resolución del problema como el proceso de evaluación en sí.

Sin embargo, debe tenerse en cuenta el factor humano y en muchos casos la sobre saturación de tecnología. Si la aplicación de una metodología de evaluación va a suponer tener que utilizar nuevas herramientas pueden dar lugar a rechazo por parte tanto de profesores como de estudiantes. En este sentido lo que se pretende con esta experiencia es facilitar la evaluación utilizando una herramienta, de especial relevancia en diferentes ámbitos de la ingeniería, que permite almacenar resultados parciales del trabajo que se está desarrollando y facilita la evaluación e interacción entre sus usuarios. Estas son las herramientas de control de versiones, Version Control System (o VCS). La idea es enseñarles a los estudiantes como utilizar una herramienta a la que van a enfrentarse en el futuro en su entorno laboral y además llevar a cabo la evaluación por pares mediante de ella.

En concreto, la presente experiencia considera la aplicación de técnicas de evaluación por pares mediante un VCS en la asignatura de Ampliación de Sistemas Operativos de segundo curso del Grado en Ingeniería Informática. Lo que se pretende es comprobar si es posible aplicar estas técnicas de evaluación usando el VCS y si su uso está relacionado con una mejora en el rendimiento del alumno en la asignatura.

En la siguiente sección se puede encontrar una descripción detallada del contexto del presente trabajo. Posteriormente, en la sección 3, se presenta cómo se aplica la evaluación por pares y cómo se compran los resultados respecto a otras anualidades. A continuación, se presentan los resultados y por último se finaliza con una serie de conclusiones.

\section{CONTEXTO}

\section{A. Evaluación por pares}

El uso de la evaluación por pares supone un cambio del modelo de evaluación tradicional, dónde la responsabilidad del aprendizaje ya no es solo del profesor sino del estudiante, que va a tener un papel más activo en la gestión de su propio aprendizaje (Fallows \& Chandramohan, 2001). Algo especialmente apreciado en el contexto educativo actual, ya que el proceso de Bolonia (European-Union, 1999) fomenta una actitud más participativa del estudiante, no solo durante la clase sino también en el proceso de evaluación.

Este tipo de planteamientos aportan al estudiante una serie de beneficios como los siguientes (Pearce, et al., 2005): 1) Mayor diversidad de perspectivas, ya que la retroalimentación respecto a su trabajo ya no solo procede del profesor, sino de sus compañeros. 2) Fomenta mayor reflexión acerca del trabajo realizado al tener disponible esa retroalimentación. 3)
Beneficios derivados de la participación en el proceso de evaluación de otros trabajos, ya que va a requerir que el alumno cuide su escritura, estudie como exponer sus ideas, aporte diferentes perspectivas, etc. 4) Desarrollo de habilidades para resolver problemas, identificar elementos críticos y aportar soluciones. 5) Incremento de la motivación y la confianza, al ser el alumno consciente del papel que tiene el proceso de evaluación. Y 6) Incremento en la independencia respecto a los "expertos", algo que puede aplicarse con posterioridad en contextos educativos y/o en entornos laborales.

Dados estos beneficios es evidente que existen multitud de iniciativas en este sentido, con aplicación en ámbitos muy diversos y con diferentes niveles de éxito (Nicol, Thomson, \& Breslin, 2014; Pastor, Pascual, \& Martín, 2005; Rodríguez Gómez, Ibarra Sáiz, \& García Jiménez, 2013; Tseng \& Tsai, 2007; van den Berg, Admiraal, \& Pilot, 2006; Xiao \& Lucking, 2008), etc.

Puesto que en este caso el ámbito es el de enseñanzas técnicas deben explorarse resultados en ese sentido, no siendo los estos tan abundantes. Algunos ejemplos pueden ser la aplicación de técnicas de evaluación por pares en asignaturas relativas a aspectos tecnológicos (o asignaturas de grados “técnicos”) (García-Pérez \& Rebollo-Catalán, 2004; Liu, Lin, Chi-Huang, \& Shyan-Ming, 2001).

Otro ejemplo, al que se debe prestar especial atención es el de la evaluación de las técnicas de evaluación por pares que se lleva a cabo en tres grados de ámbito tecnológico de la Universidad de León. En esta experiencia se observa que la aplicación de técnicas de evaluación por pares incrementa la motivación de los estudiantes y por tanto su participación y que el rendimiento de los estudiantes fue mejor con respecto a pasadas ediciones. También los estudiantes involucrados en la experiencia reflejaron que ver otras posibles soluciones, al acceder las prácticas, y obtener y dar retroalimentación de/a sus compañeros les ayuda a entender mejor los problemas y desarrollar el pensamiento crítico (Conde, Sánchez-González, Matellán-Olivera, \& Rodriguez-Lera, 2017).

Dadas estas experiencias es necesario clarificar en qué difiere el presente trabajo de otros existentes. En concreto la innovación se centra en dos factores: 1) Se trata de aplicar métodos de revisión por pares en contextos tecnológicos, existen experiencias similares, pero no que empleen herramientas no definidas de forma explícita para dicha labor; y 2) Tras la evaluación de la bibliografía realizada y desde el conocimiento de los autores no existen otras experiencias que utilicen sistemas de control de versiones para actividades de evaluación por pares.

\section{B. Sistemas de control de versiones}

En ingeniería del software, se conoce como control de versiones a la gestión de los cambios que se realizan sobre los elementos de algún producto o sobre una configuración del mismo (Fischer, Pinzger, \& Gall, 2003). A su vez, se denomina versión, revisión o edición, al estado en el que se encuentra el producto en un momento dado de su ciclo de vida. 
La gestión de versiones puede realizarse de forma manual, aunque lo recomendable es utilizar alguna herramienta para facilitar esta tarea. Estas herramientas se conocen como sistemas de control de versiones (Spinellis, 2005). Entre las más populares destacan: CVS, Subversion (Pilato, CollinsSussman, \& Fitzpatrick, 2008) o Git (Torvalds \& Hamano, 2010).

Un sistema de control de versiones debe proporcionar, como mínimo, las siguientes funcionalidades:

1. Almacenamiento para los diferentes elementos a gestionar (código fuente, imágenes, documentación).

2. Edición de los elementos almacenados (creación, borrado, modificación, renombrado, etc.).

3. Registro y etiquetado de todas las acciones realizadas, de modo que permitan devolver un elemento a un estado previo.

Dentro de los posibles sistemas de control de versiones la presente experiencia ha empleado GIT dada su popularidad y que es software libre. GIT sigue un esquema distribuido, y al contrario que otros sistemas que siguen el modelos clienteservidor, cada copia del repositorio incluye la historia completa de todos los cambios realizados (De Alwis \& Sillito, 2009).

\section{DESCRIPCIÓN}

\section{A. La asignatura}

La asignatura en la que se realiza la experiencia es “Ampliación de Sistemas Operativos" del segundo semestre de segundo curso del Grado en Ingeniería Informática. Ésta sirve como continuación de la asignatura Sistemas Operativos desarrollada en el primer semestre. En concreto, aborda el funcionamiento interno en la gestión del almacenamiento, tanto volátil (gestión de la memoria), como no volátil (gestión de archivos). También se tratan cuestiones relativas a la seguridad en los sistemas operativos.

La evaluación de la asignatura se reparte en dos bloques: la adquisición de una serie de conocimientos teóricos, que suponen un 35\% de la nota; y la realización de una serie de prácticas, que suponen el 65\% restante. Dentro del bloque práctico, uno de los trabajos que deben realizar los alumnos es la implementación de su propio sistema de ficheros. Este trabajo, supone el $65 \%$ de la nota de prácticas y es dónde se va a aplicar la evaluación por pares.

\section{B. La tarea a realizar}

Los alumnos deben implementar un sistema de ficheros basado en inodos (Rosenblum \& Ousterhout, 1992) denominado ASSOOFS (Ampliación de Sistemas Operativos File System). El sistema de ficheros debe funcionar sobre ordenadores que ejecuten el sistema operativo Linux.

Para implementar ASSOOFS los alumnos deben desarrollar un nuevo módulo para el Kernel de Linux que soporte la operación de montaje (Corbet, Rubini, \& Kroah-Hartman, 2005). ASSOOFS debe permitir la creación, la lectura y la escritura de ficheros regulares. Además, debe permitir la creación de nuevos directorios y la visualización del contenido de los directorios ya existentes.

La realización de la práctica es individual, sin embargo, para favorecer la interacción entre alumnos, el desarrollo del sistema de ficheros se realizará utilizando la plataforma GitHub que, además de incorporar el sistema de control de versiones GIT, incluye una serie de herramientas que facilitan el desarrollo colaborativo, con funcionalidades especialmente orientadas al reporte de incidencias (Issues)

Los usuarios durante la revisión por pares van a poder abrir Issues a las entregas que revisan y cerrar Issues de sus propias entregas.

\section{La evaluación por pares}

La evaluación de la práctica se realiza en tres bloques que suponen un $70 \%$, un $15 \%$ y un $15 \%$ respectivamente. La evaluación por pares se centra en el tercero de ellos:

1. Implementación de las funcionalidades básicas de ASSOFS. A saber: creación, lectura y escritura de ficheros regulares. Con respecto a la evaluación, a la nota máxima en esta parte, un 7, se aplicó una penalización de 3 puntos por errores graves, como fallos en alguna de las operaciones o errores al borrar el módulo del kernel; de 5 puntos por errores muy graves, como errores de montaje o de inserción del módulo; y de 1 punto por errores leves (permisos incorrectos, etc.)

2. Implementación de funcionalidades opcionales: creación de carpetas, cache de inodos y journaling. La implementación de alguna de las partes opcionales permitía sumar 1,5 puntos adiciones a la nota de práctica. Errores graves en esta parte suponían una penalización de un punto, y errores leves de 0,5 puntos.

3. Interacción a través de la plataforma GitHub. Esta parte incluye el uso del repositorio GIT por parte de cada alumno y la interacción con el resto a través de Issues. Para favorecer está interacción a cada alumno se le pidió que revisara el trabajo de otros 3 compañeros. Tras la revisión cada alumno debía abrir Issues con las carencias que detectadas en cada trabajo. La interacción con la plataforma se valoró en un máximo de 1,5 puntos. 0,75 de los cuales se otorgaron en base a la interacción de cada usuario con su repositorio valorándose el número de commits, el número de días en los que se realizó al menos 1 commit y el número de líneas de código fuente añadidas (Additions) y borradas (Deletions). Para el 0.75 restantes se valoró la interacción entre usuarios considerando el número de commits abiertos a otros proyectos y cerrados en el propio.

Para realizar un seguimiento de la práctica se definieron 3 hitos. En el primero, el 21 de mayo, los alumnos debían tener implementada una funcionalidad mínima que permitiera compilar e insertar y borrar el módulo compilado. Aunque no realizara las operaciones de lectura, escritura, etc., si debía mostrar trazas en el kernel cuando se intentara una de estas operaciones. 
Para el segundo hito, el 28 de mayo, los alumnos debían revisar el trabajo de sus compañeros y abrir los Issues correspondientes.

El tercer hito, el 5 de junio, se correspondía con el plazo de entrega de la práctica y era la fecha límite que tenían los alumnos para revisar y corregir los Issues abiertos por sus compañeros y terminar la funcionalidad de la práctica.

Como puede observarse la parte de la evaluación por pares tiene un peso poco significativo sobre la nota, ya que solo se corresponde con un 0,75 de la nota total, haciendo que algunos alumnos puedan prescindir de llevar a cabo esta parte. Sin embargo, el cometido de esta investigación es ver si aquellos que emplean más tiempo y tienen más actividad en la revisión por pares obtienen mejores calificaciones.

\section{Resultados}

En el presente apartado se van a mostrar los resultados para los 46 alumnos que se presentan a la asignatura respecto de los 69 matriculados, junto con sus interacciones y el factor de correlación de las actividades involucradas en la evaluación por pares con respecto a la nota final.

La tabla 1 muestra el número total de commits, el número de días en los que se hizo al menos 1 commit, el número de Additions, el número de Deletions, el número de Issues cerrados y el número de Issues abiertos por cada alumno y la nota de la práctica. De esta tabla debe destacarse que aquellos alumnos con notas más altas 8,5 son en la mayor parte de los casos los que más han participado y los que tienen mayor número de Issues cerrados y abiertos (con algunas excepciones que requirieron un estudio individual).

En la tabla 2 se muestran los promedios, desviaciones típicas de cada una de las acciones, así como de la nota. Además, para cada acción del usuario se aplica el coeficiente de correlación lineal de Pearson para ver cómo se relaciona la actividad del usuario con la nota final. Dentro de estas acciones las especialmente representativas para la evaluación por pares son los Issues Abiertos y cerrados. Cuando un estudiante abre un Issue lo hace sobre alguna de las prácticas de otros compañeros que está revisando y cuando un estudiante cierra un Issue se refiere a que ha atendido a lo que otro estudiante ha sugerido sobre su código.

En la tabla 2 pueden observarse correlaciones relativamente bajas algo que puede estar relacionado el tamaño de la muestra y con el poco peso del proceso de la evaluación por pares sobre la nota de la práctica.

La mejor correlación es entre el número de días con al menos 1 commit y la nota. Lo que implica que los estudiantes que usan más la herramienta y tienen más constancia en su trabajo obtienen mejor nota.

La segunda mejor correlación es entre el número de Issues abiertos y la nota. Lo que puede implicar que revisar el trabajo de otros ayuda a mejorar el propio, a desarrollar el pensamiento crítico y a encontrar diferentes soluciones válidas a un mismo problema. Algo que ya se había reportado en otras investigaciones (Conde, et al., 2017; Pearce, et al., 2005).
También es necesario mencionar que la tercera mejor correlación se corresponde con el número de Issues cerrados. Esto supone que atender a los problemas que otros compañeros plantean a su trabajo hacen que este mejore y por tanto la retroalimentación que estos proponen les es valiosa.

TABLA I. RESUMEN CON LA ACTIVIDAD DEL ALUMNO Y LA NOTA OBTENIDA

\begin{tabular}{|c|c|c|c|c|c|c|c|}
\hline Id & Com & $\begin{array}{l}\text { Per } \\
\text { day }\end{array}$ & Adds & Dels & $\begin{array}{c}\text { Issues } \\
\text { Cl }\end{array}$ & $\begin{array}{c}\text { Issues } \\
\text { Open }\end{array}$ & Nota \\
\hline 1 & 5 & 4 & 578 & 171 & 0 & 3 & 8,5 \\
\hline 2 & 14 & 7 & 751 & 110 & 1 & 2 & 8,5 \\
\hline 3 & 15 & 5 & 1112 & 382 & 1 & 1 & 0 \\
\hline 4 & 17 & 7 & 1812 & 662 & 1 & 3 & 6,5 \\
\hline 5 & 10 & 6 & 1457 & 424 & 1 & 1 & 0 \\
\hline 6 & 16 & 9 & 1035 & 223 & 0 & 6 & 8,5 \\
\hline 7 & 6 & 4 & 610 & 228 & 0 & 1 & 0 \\
\hline 8 & 9 & 5 & 482 & 42 & 0 & 0 & 0 \\
\hline 9 & 23 & 9 & 1431 & 880 & 0 & 2 & 8,5 \\
\hline 10 & 13 & 7 & 945 & 328 & 3 & 4 & 0 \\
\hline 11 & 13 & 9 & 1546 & 730 & 2 & 3 & 0 \\
\hline 12 & 9 & 7 & 791 & 70 & 7 & 6 & 2,5 \\
\hline 13 & 12 & 8 & 979 & 229 & 1 & 0 & 8,5 \\
\hline 14 & 18 & 9 & 1549 & 706 & 3 & 4 & 7,5 \\
\hline 15 & 11 & 8 & 821 & 114 & 0 & 2 & 7,5 \\
\hline 16 & 17 & 10 & 924 & 130 & 0 & 1 & 8,5 \\
\hline 17 & 17 & 5 & 1054 & 606 & 2 & 2 & 8 \\
\hline 18 & 9 & 6 & 513 & 64 & 3 & 2 & 8,5 \\
\hline 19 & 16 & 7 & 994 & 454 & 0 & 1 & 0 \\
\hline 20 & 9 & 6 & 1037 & 339 & 0 & 1 & 5,5 \\
\hline 21 & 24 & 12 & 4805 & 2670 & 7 & 16 & 5,5 \\
\hline 22 & 22 & 11 & 1262 & 528 & 2 & 4 & 8,5 \\
\hline 23 & 11 & 4 & 580 & 194 & 0 & 3 & 0 \\
\hline 24 & 8 & 5 & 642 & 52 & 0 & 1 & 0 \\
\hline 25 & 23 & 11 & 3171 & 1659 & 2 & 2 & 0 \\
\hline 26 & 11 & 5 & 1293 & 526 & 2 & 4 & 4,5 \\
\hline 27 & 11 & 6 & 911 & 134 & 4 & 0 & 2,5 \\
\hline 28 & 14 & 9 & 1288 & 658 & 0 & 1 & 0 \\
\hline 29 & 16 & 8 & 2460 & 522 & 1 & 1 & 4,5 \\
\hline 30 & 7 & 4 & 760 & 96 & 0 & 2 & 0 \\
\hline 31 & 3 & 3 & 116 & 38 & 0 & 0 & 0 \\
\hline 32 & 15 & 6 & 1027 & 316 & 2 & 1 & 0 \\
\hline 33 & 23 & 8 & 1332 & 2319 & 7 & 5 & 8,5 \\
\hline 34 & 17 & 9 & 1156 & 414 & 4 & 5 & 4,5 \\
\hline 35 & 5 & 3 & 533 & 68 & 0 & 3 & 0 \\
\hline 36 & 8 & 4 & 904 & 247 & 5 & 1 & 0 \\
\hline 37 & 23 & 4 & 509 & 54 & 0 & 0 & 0 \\
\hline 38 & 26 & 9 & 1003 & 377 & 0 & 0 & 0 \\
\hline 39 & 15 & 8 & 1143 & 551 & 6 & 3 & 8,5 \\
\hline 40 & 17 & 7 & 966 & 189 & 9 & 3 & 8,5 \\
\hline 41 & 18 & 9 & 1913 & 458 & 1 & 4 & 5,5 \\
\hline 42 & 7 & 4 & 954 & 90 & 1 & 3 & 8,5 \\
\hline 43 & 13 & 10 & 936 & 207 & 0 & 6 & 8,5 \\
\hline 44 & 3 & 2 & 1650 & 8 & 0 & 0 & 2,5 \\
\hline 45 & 11 & 6 & 1150 & 114 & 0 & 6 & 8,5 \\
\hline 46 & 8 & 5 & 1124 & 299 & 1 & 0 & 3,5 \\
\hline
\end{tabular}

TABLA II. DESCRIPTORES ESTADÍSTICOS Y CORRELACIÓN CON LA NOTA

\begin{tabular}{|l|c|c|c|}
\hline & Promedio & Desviación típica & Correlación \\
\hline Commits & 13,434 & 5,871 & 0,218 \\
\hline Days & 6,739 & 2,398 & 0,365 \\
\hline Additions & 1174,108 & 758,610 & 0,087 \\
\hline Deletions & 427,8267 & 536,559 & 0,119 \\
\hline Issues closed & 1,717 & 2,334 & 0,205 \\
\hline Issues opened & 2,586 & 2,720 & 0,341 \\
\hline Nota practica & 4,119 & 3,747 & \\
\hline
\end{tabular}




\section{CONCLUSIONES}

La evaluación por pares es una técnica que puede ser fácilmente aplicada en diferentes contextos de aprendizaje gracias a las TIC. En este trabajo se ha pretendido no solo llevara a cabo un caso de estudio que considere dicho tipo de evaluación sino también analizar como dicha técnica puede aplicarse mediante herramientas no pensadas como herramientas de aprendizaje, los sistemas de control de versiones. Este tipo de herramientas facilitan el desarrollo y la gestión del software, con lo que deberían ser estudiadas en cualquier grado de carácter técnico.

Al aplicar la evaluación por pares mediante un VCS se consigue por un lado que el estudiante se familiarice con este tipo de herramientas y las comience a utilizar; y por otro que se beneficie de la posibilidad de participar en un proceso de coevaluación, hecho que ha podido comprobarse a partir de las correlaciones entre las acciones de usuario relacionadas con la evaluación por pares y las notas obtenidas.

La experiencia llevada a cabo presenta diversas limitaciones. En primer lugar, el número de estudiantes involucrados debería ser mayor lo que daría lugar a unas correlaciones más fiables. En segundo lugar, el peso de la evaluación por pares (aunque coherente con otras experiencias previas) podría incrementarse para ver cómo afecta a la motivación y como esto se refleja en la nota final.

Debe mencionarse que la presente experiencia podría transferirse a otras áreas con facilidad, ya que los sistemas de control de versiones son algo que se usa en diferentes contextos, sin necesidad de que sean de ámbito tecnológico. Esto implica que su empleo como herramienta de evaluación por pares sea posible. Por ejemplo, en el ámbito de la educación se podría utilizar un sistema de gestión de versiones para distribuir el trabajo en un grupo, e ir almacenando diferentes versiones que el resto de miembros del equipo o de otros equipos podrían evaluar.

En cuanto a posibles líneas de trabajo futuro existen varias posibilidades. La primera sería comparar las notas obtenidas con respecto a ediciones anteriores de la asignatura que no emplearan evaluación por pares. También sería necesario llevar a cabo un análisis cualitativo de la experiencia, preguntando a los alumnos acerca de la evaluación y del hecho de emplear para ello el VCS, utilizando escalas como las definidas por Xiao y Lucking (2008). Además, sería interesante considerar la posibilidad de usar el VCS como herramienta no solo de evaluación por pares, sino que permita recopilar evidencias referentes al trabajo en equipo.

\section{REFERENCIAS}

Conde, M. Á., \& Hernández-García, Á. (2015). Learning analytics for educational decision making. Computers in Human Behavior, 47, 1-3. doi: http://dx.doi.org/10.1016/j.chb.2014.12.034

Conde, M. Á., Sánchez-González, L., Matellán-Olivera, V., \& Rodriguez-Lera, F. J. (2017). Application of Peer Review Techniques in Engineering Education. International Journal Engineering Education, 33(2(B)), 918-926.
Corbet, J., Rubini, A., \& Kroah-Hartman, G. (2005). Linux Device Drivers: Where the Kernel Meets the Hardware: " O'Reilly Media, Inc.".

De Alwis, B., \& Sillito, J. (2009). Why are software projects moving from centralized to decentralized version control systems? Paper presented at the Proceedings of the 2009 ICSE Workshop on cooperative and human aspects on software engineering.

European-Union. (1999). Towards the European Higher Education Area. Conference of Ministers responsible for Higher Education in 29 European countries, Bologna, Italy: Retrieved from http://www.bolognabergen2005.no/Docs/00-

Main_doc/990719BOLOGNA_DECLARATION.PDF.

Fallows, S., \& Chandramohan, B. (2001). Multiple Approaches to Assessment: Reflections on use of tutor, peer and self-assessment. Teaching in Higher Education, 6(2), 229-246. doi: 10.1080/13562510120045212

Felder, R. M., \& Silverman, L. K. (1988). Learning and teaching styles in engineering education. Engineering education, 78(7), 674-681.

Fischer, M., Pinzger, M., \& Gall, H. (2003). Populating a Release History Database from Version Control and Bug Tracking Systems. Paper presented at the Proceedings of the International Conference on Software Maintenance.

García-Pérez, R., \& Rebollo-Catalán, M. A. (2004). El modelo pedagógico de la formación universitaria y el crédito europeo: una experiencia de innovación en la asignatura Informática aplicada a la investigación educativa. RELATEC - Revista Latinoamericana de Tecnología Educativa, 3(1).

Hernández-García, Á., \& Conde, M. A. (2014). Dealing with complexity: educational data and tools for learning analytics. Paper presented at the Proceedings of the Second International Conference on Technological Ecosystems for Enhancing Multiculturality, Salamanca, Spain.

Liu, E. Z. F., Lin, S. S. J., Chi-Huang, C., \& Shyan-Ming, Y. (2001). Web-based peer review: the learner as both adapter and reviewer. Education, IEEE Transactions on, 44(3), 246-251. doi: 10.1109/13.940995

Nicol, D., Thomson, A., \& Breslin, C. (2014). Rethinking feedback practices in higher education: a peer review perspective. Assessment \& Evaluation in Higher Education, 39(1), 102-122.

O’Neill, G., \& McMahon, T. (2005). Student-centred learning: What does it mean for students and lecturers. Emerging issues in the practice of university learning and teaching I. Dublin: AISHE.

Pappano, L. (2012). The Year of the MOOC. The New York Times, 2(12).

Pastor, V. M. L., Pascual, M. G., \& Martín, J. B. (2005). La participación del alumnado en la evaluación: la autoevaluación, la coevaluación y la evaluación 
compartida. Rev. Tándem Didáctica Educ. Fís., 17, 2137.

Pearce, J., Mulder, R., \& Baik, C. (2005). Involving students in peer review: Case studies and practical strategies for university teaching. Melbourne, Australia: Centre for study on higher education (CSHE) - University of Melbourne.

Pilato, C. M., Collins-Sussman, B., \& Fitzpatrick, B. W. (2008). Version Control with Subversion: Next Generation Open Source Version Control: " O'Reilly Media, Inc.".

Rodríguez Gómez, G., Ibarra Sáiz, M., \& García Jiménez, E. (2013). Autoevaluación, evaluación entre iguales y coevaluación: conceptualización y práctica en las universidades españolas. Revista de investigación en educación, 11(2), 198-210.

Rosenblum, M., \& Ousterhout, J. K. (1992). The design and implementation of a log-structured file system. ACM Transactions on Computer Systems (TOCS), 10(1), 2652.

Savery, J. R., \& Duffy, T. M. (1995). Problem-Based Learning: An instructional model and its constructivist framework. Educational Technology, 35(5).

Spinellis, D. (2005). Version control systems. IEEE Software, 22(5), 108-109.

Topping, K. J., Smith, E. F., Swanson, I., \& Elliot, A. (2000). Formative Peer Assessment of Academic Writing Between Postgraduate Students. Assessment \& Evaluation in Higher Education, 25(2), 149-169. doi: $10.1080 / 713611428$

Torvalds, L., \& Hamano, J. (2010). Git: Fast version control system. URL http://git-scm/. com.

Tseng, S.-C., \& Tsai, C.-C. (2007). On-line peer assessment and the role of the peer feedback: A study of high school computer course. Computers \& Education, 49(4), 11611174.

doi: van den Berg, I., Admiraal, W., \& Pilot, A. (2006). Design principles and outcomes of peer assessment in higher education. Studies in Higher Education, 31(3), 341-356. doi: 10.1080/03075070600680836

van den Berg, I., Admiraal, W., \& Pilot, A. (2006). Peer assessment in university teaching: evaluating seven course designs. Assessment \& Evaluation in Higher Education, 31(1), 19-36.

Xiao, Y., \& Lucking, R. (2008). The impact of two types of peer assessment on students' performance and satisfaction within a Wiki environment. The Internet and Higher Education, 11(3-4), 186-193. doi: http://dx.doi.org/10.1016/j.iheduc.2008.06.005 